\title{
Professional nurses' perception of their clinical teaching role at a rural hospital in Lesotho
}

\author{
C N Nyoni, ${ }_{1}^{1}$ BSc Hons (Nursing Science), MSocSc (Nursing); A J Barnard, ${ }^{2}$ MB ChB, DA (SA), MFGP (SA), MPhil (Palliative Medicine)
}

${ }^{1}$ Paray School of Nursing, Thaba-Tseka, Lesotho

${ }^{2}$ Division of Family Medicine, School of Public Health and Family Medicine, Faculty of Health Sciences, University of Cape Town, South Africa

Corresponding author: C N Nyoni (cnyonioffice@gmail.com)

Background. Nursing education institutions in Lesotho face an increasing number of enrolments owing to a high demand for professional nurses to work in the community. Enrolments have doubled during the last 3 years, without an increase in teaching resources or staff. Professional nurses in the wards are expected to mentor students and teach the clinical elements of nursing while continuing with their day-to-day clinical duties. Furthermore, professional nurses in Lesotho have not been trained for this clinical teaching role.

Objective. To explore the perceptions of professional nurses with regard to their clinical teaching role in the development of competent nurses.

Methods. A qualitative study was undertaken at a rural hospital in Lesotho. Data were gathered by conducting semi-structured interviews with professional nurses. Interview transcripts were coded and emerging themes identified. Data saturation was reached after eight interview transcripts were analysed.

Results. Four themes representing the perceptions of the professional nurses emerged in the analysis: (i) the clinical teaching role; (ii) the complexities of clinical teaching; (iii) learners have their issues; and (iv) making it work.

Conclusion. Professional nurses understand and appreciate their educational role in the development of competent nurses. This clinical teaching role is performed in difficult circumstances, including administrative challenges, limited resources and staff shortages, while maintaining clinical responsibilities. Despite these challenges, the nurses have remained resilient and solution focused. Nursing education institutions should re-orientate professional nurses with regard to current trends and principles of nursing education to enhance their clinical teaching role.

Afr J Health Professions Educ 2016;8(2):166-168. DOI:10.7196/AJHPE.2016.v8i2.557

Clinical teaching is a technique used in the education of nurses. It involves the exposure of students to authentic clinical environments and guiding them to attain specific requirements applicable in that particular environment. ${ }^{[1]}$ The clinical environment is a platform for the application of knowledge and skills that have already been introduced in the classroom. Nursing students in the clinical environment integrate knowledge and skills learnt in a contextualised learning environment, which should support them in their educational objectives. ${ }^{[2]}$ Competent professional nurses could enhance the quality of the clinical environment as a learning platform.

The competency of the professional nurse in the clinical environment is essential for the success of the nursing student. They should possess attributes that enhance clinical teaching. ${ }^{[3]}$ Some of the attributes include the ability to identify brief periods during which to teach students, awareness of their own professional role and responsibility, and ability to manage the teaching platform for the development of the nursing student. If professional nurses are aware of their professional role in the clinical environment, it enhances their functionality.

A review of the literature highlights that professional nurses are involved in clinical teaching, which is conducted through various models, depending on the context or setting. Models used in clinical teaching include: apprenticeship, internship, supervision, and preception. ${ }^{[4]}$ Professional nurses tend to move towards the supervisory model of clinical teaching, especially for students in basic nursing or pre-registration programmes. This model involves the allocation of nursing students to a qualified professional nurse who, in the course of clinical practice, shares knowledge and skills with the students. However, while in the clinical area, students are expected to observe and absorb the lessons learnt while participating in activities overseen by a qualified professional nurse. The learning includes knowledge and skills that may be acquired in that context, to aspects of professional practice and organisational culture.

The healthcare delivery system in Lesotho is led by nurses, and the majority of healthcare professionals trained there are nurses. The number of professional nurses in Lesotho is inadequate to meet the burden of disease. The government of Lesotho has intervened, through its partners, to increase the number of nurses completing training in the country. This has increased the enrolment of student nurses since 2010.

This increased enrolment translates into increased student numbers in the clinical setting at any given time. These increased numbers and the demands of nursing education institutions have led to a degree of role confusion among professional nurses. Professional role confusion may be associated with changes in the workplace, and influence regulatory authorities and the nursing education institutions' expectations of professionals in the clinical environment. ${ }^{[5]}$ Professional role confusion may affect the quality of clinical teaching and subsequently student outcomes. This article describes the perceptions of professional nurses with regard to their clinical teaching role at a rural hospital in Lesotho.

\section{Methods}

This was a descriptive qualitative study and included professional nurses who qualified in general nursing and midwifery at a rural hospital in Lesotho. Eight professional nurses who had previously worked with student nurses in the clinical environment were purposively selected. Data were obtained from semi-structured interviews conducted by one of the researchers, using an interview guide. The interviews explored the professional nurses' perceptions and experiences of teaching nursing students in the clinical environment. Data saturation was reached after eight semi-structured interviews. 
The audio-recorded interviews were transcribed and coded to display themes that reflected the perceptions of the professional nurses with regard to their clinical teaching role. Consensus on the themes that emerged from the data analysis was reached between the researcher and an expert. The trustworthiness of the study was further enhanced by means of member checking. The participants confirmed the accuracy of the results and that these represented their perceptions. ${ }^{[6]}$

Ethical approval was granted by the Institutional Review Board of the Ministry of Health of Lesotho (REC 05/2013). Access to the rural hospital was granted by the manager, Hospital Nursing Services, on behalf of the hospital board.

\section{Results}

Analysis of the transcripts of the semi-structured interviews with the professional nurses generated four themes. These include: (i) the clinical teaching role; (ii) the complexities of clinical teaching; (iii) learners have their issues; and (iv) making it work.

The description of the results is structured around these themes and is supported by some quotes from the interviews.

\section{Theme 1: The clinical teaching role}

Professional nurses felt an obligation to teach student nurses in the clinical environment. The former emphasised that they have to interact with students while attending to the day-to-day running of the ward. The following quote demonstrates this dual role:

'... when students are in the wards, I know it is expected of me to teach them what I know. (NF3)

This statement shows that the professional nurses understand that their role includes clinical teaching. This is also supported in the literature, which discusses the dual working role of professional nurses as clinical practitioners and clinical teachers in the working environment. ${ }^{[2]}$

Even though professional nurses expect that their clinical positions encompass a teaching role, they feel that there is no consultation or preparation for clinical teaching. They complain that they are incorporated into the training of students without their opinions or challenges being taken into consideration. The following quote illustrates the perception of a professional nurse who was propelled into a teaching role in the clinical situation, with insufficient preparation and consequent denial of identification as a teacher:

'Just when I got employed ... I see students and I am supposed to teach them ... I was reminded that it's part of my job but I don't know what and how to teach them ... I am not a teacher ... ? (NM1)

The expectation that professional nurses facilitate clinical learning of nursing students imposes an added burden on these busy clinical practitioners. This was felt to have been 'thrust upon them'. One positive response to the added burden was to integrate the clinical teaching role with all their daily tasks. Models of clinical teaching that nurses recalled from their own training formed the basis of their clinical teaching practice, as illustrated in the next quote:

'I recognise that I have an extra load of work when the students are around, we have to be busy and include them in whatever we do but me I just teach them the way we were taught ... ? (NF8)

\section{Theme 2: The complexities of clinical teaching}

Statements grouped under this theme reflected the complexity of the clinical teaching process in the context of a rural hospital. Students should be afforded the best learning opportunities in an authentic environment. The professional nurses highlighted some of the complexities and challenges that hampered the process of clinical teaching:

'At times I really wish these students could actually practise the real thing ... it's disheartening to always compromise the quality of training because we are out of supplies.' (NF4)

This quote above shows that the unavailability of everyday resources hampers the clinical teaching process. ${ }^{[4]}$ These challenges reduced the effectiveness of professional nurses as clinical teachers. The nurses highlighted that the resource constraints widened the theory-practice gap. It is clear from the literature that the theory-practice gap affects the overall process of learning. ${ }^{[7]}$ This phenomenon may be considered from the point of view of the classroom, where the curriculum is not based on the healthcare delivery system; or from the point of view of the clinical learning site, where the students' learning experiences are not aligned with the requirements of the curriculum. The following shows that this mismatch is present in practice:

'... some of the things that they wish to do are not practical here, so we tell them to practise what and how we do it here ... [not] as it is demonstrated at school.' (NF3)

The increased student enrolment was challenging and made clinical teaching difficult. However, increased student numbers offered relief from some clinical tasks, as the students would do some of the work in the wards:

'I am excited when I hear that we are having students here, I know someone has to do some of my work, so I sit back and relax.' (NM1)

\section{Theme 3: Learners have their issues}

The trainees, in this case the nursing students in the clinical environment, contribute to the realisation of the clinical teaching role of the professional nurse. The latter explained that a dynamic relationship of trust between trainer and trainee is essential for teaching and learning to be effective, and for the commitment and objectives of the trainees to be demonstrated:

'These learners have to be treated on an individual basis. After spending a day or two with me, I can tell who is interested in their work or not.' (NF6)

According to the professional nurses, the student nurses did not seem to be aware of their own responsibilities or of the purpose of clinical placement. Nursing students failed to identify their own learning needs and had poor levels of self-motivation. The professional nurses indicated that they had limited skills in identifying teaching opportunities. Should students fail to take the initiative, they are forgotten in the day-to-day running of the wards. This lack of initiative proved to be a barrier to training. The following quote demonstrates this aspect, but may also indicate that there are further student issues that require investigation:

'The students that are attached to my ward are usually many and that may lead to over-crowding and it is in this over-crowding that a student who does not talk disappears due to silence, I mean I forget that she is there.' (NF7)

\section{Theme 4: Making it work}

It was recognised that clinical teaching is an important activity that has been carried out by professional nurses in the ward for many years. The professional nurses confirmed that they are aware of their role in training and developing students during the time that they are in the wards, despite 
operational difficulties. The professional nurses suggested solutions that could enhance their clinical teaching role, including continuing professional development aimed at keeping them up to date with clinical practice:

'We need to be put in the loop when it comes to [the] latest information with regards to disease and new evidence, ... technical assistance and other new information on patient management routinely from the school ... ? (NF5)

They suggested that professional development should include academic support and motivation. They would prefer their clinical teaching role to be appreciated more. Commitment from stakeholders in nursing education, especially the employers, was mentioned as critical for the effectiveness of professional development aimed at improving their training role:

'If we are to work together towards these refresher courses, I would like my employer (hospital) to be part of it and support me throughout.' (NM1)

\section{Discussion}

The literature shows that there is a shortage of nursing faculty in many African countries $^{[8]}$ and that professional nurses are the clinical teachers for student nurses in the wards. The findings of this study support those of previous research that explored the experiences and perceptions of healthcare workers in clinical environments about the training of students. ${ }^{[5,9]}$

Nursing education institutions use professional nurses working in the clinical environment to train nursing students, particularly in the acquisition of skills and in integrating theory and practice. It is essential that these professional nurses have some degree of experience and an awareness of their clinical teaching role. This is intended to enable authentic and contextual learning, representative of clinical practice in the real world, thereby better preparing the students for practice.

Professional nurses are expected to adopt both clinical and training roles, despite their relative inexperience in both areas. These findings were consistent with the experience in other rural training institutions in Africa. ${ }^{[9]}$ Owing to the rural nature of the setting, the more experienced professional nurses moved to urban areas and some leave the country, leaving only young and inexperienced professionals. These novices require further mentorship and guidance. ${ }^{[10]}$ They are expected to work in clinical settings and carry the burden of orientating and training students. ${ }^{[1]}$

The facilitation of skills development, critical thinking and clinical judgement is an important task that requires training. The professional nurses identified the need for training in 'learning facilitation' as a critical gap in their preparation for a clinical teaching role. The curriculum for training general nurses and midwives is practice orientated, with no elements of nursing education. The lack of knowledge in the basic principles of clinical teaching reduces the effectiveness of the professional nurse in identifying brief periods for teaching, assessing students fairly and giving feedback and debriefing sessions. ${ }^{[9,12]}$ These aspects of nursing education are critical for all professional nurses, particularly in Africa, where they all have a direct clinical teaching role. ${ }^{[9]}$

The professional nurse is expected to provide skilled guidance to students, as they apply classroom theory in practice. This study found that professional nurses perceive themselves as fitting in the educational process of student nurses. The clinical teaching role is performed in the context of operational difficulties, which are either beyond the control of the professional nurse, such as lack of resources, or within the control of the nurse, such as personal interest and a lack of a sense of lifelong learning. Lifelong learning is an important attribute of all health professionals that enables them to actively search for information when necessary. The nurses recognised that they have knowledge gaps that often made it difficult for them to teach students. As one of the professional nurses said, '.. it is very difficult to teach whatever you don't know'.

Nursing education institutions have high expectations of professional nurses with regard to their clinical teaching role. Despite the limited resources, professional nurses have shown resilience and willingness to find solutions to the problems experienced. The study showed that professional nurses had some suggestions to improve their clinical teaching roles. At the rural hospital they reflected on their strengths and weaknesses and recommended continued professional development in the clinical environment as a strategy to enhance clinical teaching. Such a strategy should address the actual and perceived knowledge gaps among practising nurses. The stakeholders of nursing education in Lesotho should demonstrate commitment to the continued training and professional development of professional nurses in clinical environments, including their clinical teaching roles.

Future areas of research should explore the training needs of nurses with regard to clinical teaching and lifelong learning needs. This could inform the development of continuing professional development strategies.

This study was carried out at a rural hospital in Lesotho and the findings cannot be generalised to the other training hospitals in Lesotho or elsewhere. Although this may be viewed as a study limitation, the results highlight the perspective of healthcare workers in rural institutions who are expected to perform roles for which they are not suitably trained.

\section{Conclusion}

We recommend that nursing education institutions work in collaboration with teaching hospitals to devise and implement continuing professional development programmes to address the nursing education needs of professional nurses. Support strategies should be established for newly qualified professional nurses to assist them in their clinical teaching role. This support should be extended to all professional nurses in the hospital. Continuing enhancement of skills of professional nurses who are working with students is essential to educate competent nurses.

\section{References}

1. Spencer J. Learning and teaching in the clinical environment. BMJ 2003;326(7389):591. DOI:10.1136/bmj.326.7389.591 2. Botma Y, Herter S, Kotze R. Responsibilities of nursing schools with regard to peer mentoring. Nurse Educ Today 2013;33(8):808-813. DOI:10.1016/.jnedt.2012.02.21

3. Jokelainen M, TurumenH, Tossavainen K, Jamookeeah D, Coco K. A systematic review of mentoring nursing students in clinical placements. J Clin Nurs 2011;20(19-20):2854-2867. DOI:101111/j.365-2702.2010.03571.x 4. Gaberson KB, Oermann MH, Shellenbarger T. Clinical Teaching Strategies in Nursing. 4th ed. New York Springer, 2014.

5. Bray L, Nettleton P. Assessor or mentor: Role confusion in professional education. Nurse Educ Today 2007;27(8):848-855. DOI:10.1016/.nedt2006.11006

6. De Vos AS, Stryd He Herial Sciences and Hum 6. De Vos AS, Strydom H, Fouche CB, Delport CSL. Resear
Services Professions. 4th ed. Pretoria: Van Schaik, 2011.

7. Hervices Pofessions. Hh De. Pretona: Van Schak, 2011. Houghton CE, Casey D, Shaw D, Murphy K. Students' experiences of implemen
world of practice. J Clin Nurs 2013;22(13-14):1961-1969. DOI:10.1111/jocn.12014

8. Sherr K, Mussa A, Chilundo B, et al. Brain drain and health workforce distortion in Mozambique. PLoS One 2012;7(4):e35840. DOI:10.1371/journal.pone.0035840

9. Eta VEA, Atanga MBS, Atashili J, D’Cruz G. Nurses and challenges faced as clinical educators: A survey of a group of nurses in Cameroon. Pan Afr Med J 2011;8(1):28. DOI:10.4314/pamj.v811.71085

10. Benner P. From novice to expert. Am J Nurs 1982; 2(3):402-407. DOI:10.2307/3462928

11. Ogochukwu CG, Uys LR, Karani AK, Okoronkwo IL, Diop BN. Roles of nurses in sub-Saharan African region. Int J Nurs Midwif 2013;5(7):117-131. DOI:10.5897/ijnm2013.0104

12. Makhakhe AM. Nurses' experience of the transition from student to professional practitioner in a public hospital in Lesotho. MCur thesis. Potchefstroom: North West University, 2011. 